\title{
Algorithmic Approach to the Equilibrium Points and Fixed Points
}

\author{
Lijin Guo, ${ }^{1}$ Shin Min Kang, ${ }^{2}$ and Young Chel Kwun ${ }^{3}$ \\ ${ }^{1}$ School of Electrical Engineering and Automation, Tianjin Polytechnic University, Tianjin 300387, China \\ ${ }^{2}$ Department of Mathematics and the RINS, Gyeongsang National University, Jinju 660-701, Republic of Korea \\ ${ }^{3}$ Department of Mathematics, Dong-A University, Pusan 614-714, Republic of Korea
}

Correspondence should be addressed to Young Chel Kwun; yckwun@dau.ac.kr

Received 10 March 2014; Accepted 25 March 2014; Published 14 April 2014

Academic Editor: Chong Li

Copyright (c) 2014 Lijin Guo et al. This is an open access article distributed under the Creative Commons Attribution License, which permits unrestricted use, distribution, and reproduction in any medium, provided the original work is properly cited.

The equilibrium and fixed point problems are considered. An iterative algorithm is presented. Convergence analysis of the algorithm is provided.

\section{Introduction}

Let $H$ be a real Hilbert space with inner product $\langle\cdot, \cdot\rangle$ and norm $\|\cdot\|$, respectively. Let $C$ be a nonempty closed convex subset of $H$. Let $\Phi: C \rightarrow H$ be a nonlinear operator and let $\Theta: C \times C \rightarrow R$ be a bifunction. The equilibrium problem is formulated as finding $x^{\dagger} \in C$ such that

$$
\Theta\left(x^{\dagger}, x\right)+\left\langle\Phi\left(x^{\dagger}\right), x-x^{\dagger}\right\rangle \geq 0, \quad \forall x \in C .
$$

The solution set of (1) is denoted by EP. The problem (1) is very general in the sense that it includes, as special cases, optimization problems, variational inequalities, minimax problems, Nash equilibrium problem in noncooperative games, and others. For related work, please see, for example, [1-17]. Next, we recall several interesting results where $\Theta$ verifies the following usual conditions $(C 1)-(C 4)$ which will be used in the sequel:

(C1) $\Theta(u, u)=0$ for all $u \in C$;

$(C 2) \Theta$ is monotone; that is, $\Theta(u, v)+\Theta(v, u) \leq 0$ for all $u, v \in C$;

(C3) for each $u, v, w \in C, \lim _{t \downarrow 0} \Theta(t w+(1-t) u, v) \leq \Theta(u, v)$;

(C4) for each $u \in C, v \mapsto \Theta(u, v)$ is convex and lower semicontinuous.
Theorem 1. Let $C$ be a nonempty closed convex subset of $H$. Let $\Theta: C \times C \rightarrow R$ be a bifunction which satisfies conditions (C1)-(C4). Let $S: C \rightarrow C$ be a nonexpansive mapping. Let $\phi: H \rightarrow H$ be a contraction. For $x_{0} \in H$ arbitrarily, let the sequence $\left\{x_{n}\right\}$ be generated by

$$
\begin{gathered}
\Theta\left(y_{n}, x^{\dagger}\right)+\frac{1}{\lambda_{n}}\left\langle x^{\dagger}-y_{n}, y_{n}-x_{n}\right\rangle \geq 0, \quad \forall x^{\dagger} \in C, \\
x_{n+1}=\alpha_{n} \phi\left(x_{n}\right)+\left(1-\alpha_{n}\right) S y_{n}, \quad n \geq 0,
\end{gathered}
$$

where $\left\{\alpha_{n}\right\} \subset[0,1]$ and $\left\{\lambda_{n}\right\} \subset(0, \infty)$ satisfy $\lim _{n \rightarrow \infty} \alpha_{n}=0$, $\sum_{n=1}^{\infty} \alpha_{n}=\infty, \sum_{n=1}^{\infty}\left|\alpha_{n+1}-\alpha_{n}\right|<\infty, \liminf _{n \rightarrow \infty} \lambda_{n}>0$, and $\sum_{n=1}^{\infty}\left|\lambda_{n+1}-\lambda_{n}\right|<\infty$. Then, the sequence $\left\{x_{n}\right\}$ converges strongly to $x^{\ddagger}=P_{F(S) \cap E P} \phi\left(x^{\ddagger}\right)$ provided $F(S) \cap E P \neq \emptyset$.

Chuang et al. [18] considered an iteration process of Halpern's type for finding a common element of the set of solutions of an equilibrium problem and the set of fixed points for a nonexpansive mapping with perturbation in a Hilbert space and they proved a strong convergence theorem for such iterations.

Theorem 2. Let $C$ be a nonempty closed convex subset of $H$. Let $\Theta: C \times C \rightarrow R$ be a bifunction which satisfies conditions (C1)-(C4). Let $S: C \rightarrow H$ be a nonexpansive mapping. Let 
$\left\{u_{n}\right\} \subset H$ be a sequence. For $x_{0} \in H$ arbitrarily, let the sequence $\left\{x_{n}\right\}$ be generated by

$$
\begin{gathered}
\Theta\left(y_{n}, x^{\dagger}\right)+\frac{1}{\lambda_{n}}\left\langle x^{\dagger}-y_{n}, y_{n}-x_{n}\right\rangle \geq 0, \quad \forall x^{\dagger} \in C, \\
x_{n+1}=\alpha_{n} u_{n}+\left(1-\alpha_{n}\right)\left(\beta_{n} y_{n}+\left(1-\beta_{n}\right) S y_{n}\right), \quad n \geq 0,
\end{gathered}
$$

where $\left\{\alpha_{n}\right\} \subset[0,1]$ and $\left\{\lambda_{n}\right\} \subset[a, \infty)$ satisfy $\lim _{n \rightarrow \infty} \alpha_{n}=0$, $\sum_{n=1}^{\infty} \alpha_{n}=\infty, \lim _{n \rightarrow \infty} \beta_{n}\left(1-\beta_{n}\right)>0$, and $\lim _{n \rightarrow \infty} u_{n}=$ $u \in H$. Then, the sequence $\left\{x_{n}\right\}$ converges strongly to $x^{\ddagger}=$ $P_{F(S) \cap E P}(u)$ provided $F(S) \cap E P \neq \emptyset$.

S. Takahashi and W. Takahashi [17] introduced the following iterative algorithm for finding an element of $F(S) \cap E P$ :

$$
\begin{gathered}
\Theta\left(y_{n}, x^{\dagger}\right)+\left\langle\Phi\left(x_{n}\right), x^{\dagger}-y_{n}\right\rangle \\
+\frac{1}{\lambda_{n}}\left\langle x^{\dagger}-y_{n}, y_{n}-x_{n}\right\rangle \geq 0, \quad \forall x^{\dagger} \in C, \\
x_{n+1}=\beta_{n} x_{n}+S\left[\alpha_{n} u+\left(1-\beta_{n}\right) y_{n}\right], \quad n \geq 0 .
\end{gathered}
$$

And they proved that the sequence $\left\{x_{n}\right\}$ converges strongly to $x^{\ddagger}=P_{F(S) \cap E P}(u)$.

Remark 3. Algorithm (3) is involved in a variant anchor $\left\{u_{n}\right\}$ and the parameters are also relaxed. In [17], the authors considered a general equilibrium problem.

In this paper, our main purpose is to introduce a new iteration process for finding a common element of the set of solutions of an equilibrium problem and the set of fixed points for a nonexpansive mapping in a Hilbert space and then we prove a strong convergence theorem for such iterations. Our iterations are very different from (2)-(4). As a special case, we can find the minimum norm solution of $F(S) \cap \mathrm{EP}$.

\section{Preliminaries}

In the sequel, we assume $H$ is a real Hilbert space. Let $C \subset H$ be a nonempty closed convex set. Recall that a mapping $\Phi$ : $C \rightarrow H$ is called $\alpha$-inverse-strongly monotone if there exists a positive real number $\alpha>0$ such that

$$
\langle\Phi(u)-\Phi(v), u-v\rangle \geq \alpha\|\Phi(u)-\Phi(v)\|^{2}, \quad \forall u, v \in C .
$$

A mapping $S: C \rightarrow C$ is said to be nonexpansive if

$$
\|S u-S v\| \leq\|u-v\|, \quad \forall u, v \in C .
$$

We use $F(T)$ to denote the set of fixed points of $S$.

The following lemmas are useful for the next section.

Lemma 4 (see [11]). Let $C$ be a nonempty closed convex subset of $H$. Let $\Theta: C \times C \rightarrow R$ be a bifunction which satisfies conditions (C1)-(C4). Let $\lambda>0$ and $x \in H$. Then, there exists $y \in C$ such that

$$
\Theta\left(y, x^{\dagger}\right)+\frac{1}{\lambda}\left\langle x^{\dagger}-y, y-x\right\rangle \geq 0, \quad \forall x^{\dagger} \in C .
$$

Set $T_{\lambda}(x)=\left\{y \in C: \Theta\left(y, x^{\dagger}\right)+(1 / \lambda)\left\langle x^{\dagger}-y, y-x\right\rangle \geq\right.$ 0 , for all $\left.x^{\dagger} \in C\right\}$. Then the following hold:

(i) $T_{\lambda}$ is single-valued and $T_{\lambda}$ is firmly nonexpansive; that is for any $x, y \in H,\left\|T_{\lambda} x-T_{\lambda} y\right\|^{2} \leq\left\langle T_{\lambda} x-T_{\lambda} y, x-y\right\rangle$;

(ii) EP is closed and convex and $E P=F\left(T_{\lambda}\right)$.

Lemma 5. Let $C, H, F$, and $T_{\lambda} x$ be as in Lemma 4. Then the following holds:

$$
\left\|T_{\lambda} x-T_{\gamma} x\right\|^{2} \leq \frac{\lambda-\gamma}{\lambda}\left\langle T_{\lambda} x-T_{\gamma} x, T_{\lambda} x-x\right\rangle
$$

for all $\lambda, \gamma>0$ and $x \in H$.

Lemma 6 (see [19]). Let $C$ be a nonempty closed convex subset of $H$. Let the mapping $\Phi: C \rightarrow H$ be $\alpha$-inverse-strongly monotone and let $\lambda>0$ be a constant. Then, we have

$$
\begin{aligned}
& \|(I-\lambda \Phi) u-(I-\lambda \Phi) v\|^{2} \\
& \quad \leq\|u-v\|^{2}+\lambda(\lambda-2 \alpha)\|\Phi(u)-\Phi(v)\|^{2}, \quad \forall u, v \in C .
\end{aligned}
$$

In particular, if $0 \leq \lambda \leq 2 \alpha$, then $I-\lambda \Phi$ is nonexpansive.

Lemma 7 (see [20]). Let $C$ be a closed convex subset of $H$ and let $S: C \rightarrow C$ be a nonexpansive mapping with $F(S) \neq \emptyset$. Then, the mapping $I-S$ is demiclosed. That is, if $\left\{x_{n}\right\}$ is a sequence in $C$ such that $x_{n} \rightarrow x^{*}$ weakly and $(I-S) x_{n} \rightarrow y$ strongly, then $(I-S) x^{*}=y$.

Lemma 8 (see [21]). Let $\left\{R_{n}\right\}$ and $\left\{W_{n}\right\}$ be two bounded sequences in $H$. Let $\left\{\beta_{n}\right\}$ be a sequence in $[0,1]$ satisfying $0<$ $\liminf _{n \rightarrow \infty} \beta_{n} \leq \limsup _{n \rightarrow \infty} \beta_{n}<1$. Suppose that $R_{n+1}=$ $\left(1-\beta_{n}\right) W_{n}+\beta_{n} R_{n}$ for all $n \geq 0$ and $\limsup _{n \rightarrow \infty}\left(\| W_{n+1}-\right.$ $\left.W_{n}\|-\| R_{n+1}-R_{n} \|\right) \leq 0$. Then, $\lim _{n \rightarrow \infty}\left\|W_{n}-R_{n}\right\|=0$.

Lemma 9 (see [22]). Assume that $\left\{a_{n}\right\}$ is a sequence of nonnegative real numbers such that

$$
a_{n+1} \leq\left(1-\gamma_{n}\right) a_{n}+\delta_{n} \gamma_{n}
$$

where $\left\{\gamma_{n}\right\}$ is a sequence in $(0,1)$ and $\left\{\delta_{n}\right\}$ is a sequence such that

(1) $\sum_{n=1}^{\infty} \gamma_{n}=\infty$;

(2) $\lim \sup _{n \rightarrow \infty} \delta_{n} \leq 0$ or $\sum_{n=1}^{\infty}\left|\delta_{n} \gamma_{n}\right|<\infty$.

Then $\lim _{n \rightarrow \infty} a_{n}=0$.

\section{Main Results}

In this section, we will prove our main results.

Theorem 10. Let $C$ be a nonempty closed convex subset of $H$ and let $\Theta: C \times C \rightarrow R$ be a bifunction satisfying conditions (C1)-(C4). Let $\Phi: C \rightarrow H$ be an $\varsigma$-inverse-strongly monotone mapping and let $S: C \rightarrow C$ be a nonexpansive mapping. 
Suppose that $F(S) \cap E P \neq \emptyset$. Let $\left\{u_{n}\right\}$ be a sequence in $H$. For $x_{0} \in C$ arbitrarily, let the sequence $\left\{x_{n}\right\}$ be generated by

$$
\begin{aligned}
x_{n+1}=\omega_{n} x_{n}+\left(1-\omega_{n}\right) S T_{\rho_{n}} & {\left[\left(1-\varsigma_{n}\right) x_{n}\right.} \\
& \left.+\varsigma_{n} u_{n}-\rho_{n} \Phi\left(x_{n}\right)\right], \quad n \geq 0,
\end{aligned}
$$

where $T_{\rho_{n}}$ is defined as that in Lemma 4 and $\left\{\rho_{n}\right\} \quad C$ $(0,2 \varsigma),\left\{\varsigma_{n}\right\} \subset(0,1)$, and $\left\{\omega_{n}\right\} \subset(0,1)$ satisfy

(i) $\lim _{n \rightarrow \infty} \varsigma_{n}=0$ and $\sum_{n=1}^{\infty} \varsigma_{n}=\infty$;

(ii) $0<c \leq \omega_{n} \leq d<1$;

(iii) $a\left(1-\varsigma_{n}\right) \leq \rho_{n} \leq b\left(1-\varsigma_{n}\right)$, where $[a, b] \subset(0,2 \varsigma)$ and $\lim _{n \rightarrow \infty}\left(\rho_{n+1}-\rho_{n}\right)=0$;

(iv) $\lim _{n \rightarrow \infty} u_{n}=u$.

Then $\left\{x_{n}\right\}$ generated by (11) converges strongly to $P_{F(S) \cap E P}(u)$.

We divide our proofs into several conclusions.

Conclusion 1. The sequence $\left\{x_{n}\right\}$ is bounded.

Proof. Let $z \in F(S) \cap$ EP. We have $z=S z=T_{\rho_{n}}\left(z-\rho_{n} \Phi(z)\right)=$ $T_{\rho_{n}}\left[\varsigma_{n} z+\left(1-\varsigma_{n}\right)\left(z-\rho_{n} \Phi(z) /\left(1-\varsigma_{n}\right)\right)\right]$ for all $n \geq 0$. Set $z_{n}=T_{\rho_{n}}\left[\left(1-\varsigma_{n}\right) x_{n}+\varsigma_{n} u_{n}-\rho_{n} \Phi\left(x_{n}\right)\right]$ for all $n \geq 0$. By Lemma 4 , we know that $T_{\rho_{n}}$ is nonexpansive. By the convexity of $\|\cdot\|$, we derive

$$
\begin{aligned}
& \left\|z_{n}-z\right\|^{2}=\| T_{\rho_{n}}\left[\left(1-\varsigma_{n}\right) x_{n}+\varsigma_{n} u_{n}-\rho_{n} \Phi\left(x_{n}\right)\right] \\
& -T_{\rho_{n}}\left(z-\rho_{n} \Phi(z)\right) \|^{2} \\
& =\| T_{\rho_{n}}\left[\varsigma_{n} u_{n}+\left(1-\varsigma_{n}\right)\left(x_{n}-\frac{\rho_{n}}{1-\varsigma_{n}} \Phi\left(x_{n}\right)\right)\right] \\
& -T_{\rho_{n}}\left[\varsigma_{n} z+\left(1-\varsigma_{n}\right)\left(z-\frac{\rho_{n}}{1-\varsigma_{n}} \Phi(z)\right)\right] \|^{2} \\
& \leq \|\left[\varsigma_{n} u_{n}+\left(1-\varsigma_{n}\right)\left(x_{n}-\frac{\rho_{n}}{1-\varsigma_{n}} \Phi\left(x_{n}\right)\right)\right] \\
& -\left[\varsigma_{n} z+\left(1-\varsigma_{n}\right)\left(z-\frac{\rho_{n}}{1-\varsigma_{n}} \Phi(z)\right)\right] \|^{2} \\
& =\|\left(1-\varsigma_{n}\right)\left[\left(x_{n}-\frac{\rho_{n}}{1-\varsigma_{n}} \Phi\left(x_{n}\right)\right)\right. \\
& \left.-\left(z-\frac{\rho_{n}}{1-\varsigma_{n}} \Phi(z)\right)\right]+\varsigma_{n}\left(u_{n}-z\right) \|^{2} \\
& \leq\left(1-\varsigma_{n}\right) \|\left(x_{n}-\frac{\rho_{n}}{1-\varsigma_{n}} \Phi\left(x_{n}\right)\right) \\
& -\left(z-\frac{\rho_{n}}{1-\varsigma_{n}} \Phi(z)\right)\left\|^{2}+\varsigma_{n}\right\| u_{n}-z \|^{2} .
\end{aligned}
$$

Since $\Phi$ is $\varsigma$-inverse-strongly monotone, we know from Lemma 6 that

$$
\begin{aligned}
& \left\|\left(x_{n}-\frac{\rho_{n}}{1-\varsigma_{n}} \Phi\left(x_{n}\right)\right)-\left(z-\frac{\rho_{n}}{1-\varsigma_{n}} \Phi(z)\right)\right\|^{2} \\
& \quad \leq\left\|x_{n}-z\right\|^{2}+\frac{\rho_{n}\left(\rho_{n}-2\left(1-\varsigma_{n}\right) \varsigma\right)}{\left(1-\varsigma_{n}\right)^{2}}\left\|\Phi\left(x_{n}\right)-\Phi(z)\right\|^{2} .
\end{aligned}
$$

It follows that

$$
\begin{gathered}
\left\|z_{n}-z\right\|^{2} \leq\left(1-\varsigma_{n}\right)\left(\left\|x_{n}-z\right\|^{2}+\frac{\rho_{n}\left(\rho_{n}-2\left(1-\varsigma_{n}\right) \varsigma\right)}{\left(1-\varsigma_{n}\right)^{2}}\right. \\
\left.\times\left\|\Phi\left(x_{n}\right)-\Phi(z)\right\|^{2}\right)+\varsigma_{n}\left\|u_{n}-z\right\|^{2}, \\
\leq\left(1-\varsigma_{n}\right)\left\|x_{n}-z\right\|^{2}+\varsigma_{n}\left\|u_{n}-z\right\|^{2} .
\end{gathered}
$$

So, we have that

$$
\begin{aligned}
\left\|x_{n+1}-z\right\|^{2}= & \left\|\omega_{n}\left(x_{n}-z\right)+\left(1-\omega_{n}\right)\left(S z_{n}-z\right)\right\|^{2} \\
\leq & \omega_{n}\left\|x_{n}-z\right\|^{2}+\left(1-\omega_{n}\right)\left\|z_{n}-z\right\|^{2} \\
\leq & \omega_{n}\left\|x_{n}-z\right\|^{2}+\left(1-\omega_{n}\right)\left(\left(1-\varsigma_{n}\right)\left\|x_{n}-z\right\|^{2}\right. \\
& \left.+\varsigma_{n}\left\|u_{n}-z\right\|^{2}\right) \\
= & {\left[1-\left(1-\omega_{n}\right) \varsigma_{n}\right]\left\|x_{n}-z\right\|^{2} } \\
& +\left(1-\omega_{n}\right) \varsigma_{n}\left\|u_{n}-z\right\|^{2} \\
\leq & \max \left\{\left\|u_{n}-z\right\|^{2},\left\|u_{n}-z\right\|^{2}\right\} .
\end{aligned}
$$

Note that $\lim _{n \rightarrow \infty} u_{n}=u \in H$. Without loss of generality, we can assume that $\sup _{n}\left\|u_{n}-u\right\| \leq M$ for some $M>0$.

By induction, we have

$$
\begin{aligned}
\left\|x_{n+1}-z\right\|^{2} & \leq \max \left\{\left\|x_{0}-z\right\|^{2},\left(\left\|u_{n}-u\right\|+\|u-z\|\right)^{2}\right\} \\
& \leq \max \left\{\left\|x_{0}-z\right\|^{2},(M+\|u-z\|)^{2}\right\} .
\end{aligned}
$$

Therefore, $\left\{x_{n}\right\}$ is bounded.

Conclusion 2. $\lim _{n \rightarrow \infty}\left\|x_{n+1}-x_{n}\right\|=0$ and $\lim _{n \rightarrow \infty} \| x_{n}-$ $T_{\rho_{n}}\left[\left(1-\varsigma_{n}\right) x_{n}+\varsigma_{n} u_{n}-\rho_{n} \Phi\left(x_{n}\right)\right] \|=0$.

Proof. Putting $w_{n}=\left(1-\varsigma_{n}\right) x_{n}+\varsigma_{n} u_{n}-\rho_{n} \Phi\left(x_{n}\right)$ for all $n \geq 0$, we have

$$
z_{n+1}-z_{n}=T_{\rho_{n+1}} w_{n+1}-T_{\rho_{n+1}} w_{n}+T_{\rho_{n+1}} w_{n}-T_{\rho_{n}} w_{n} .
$$

It follows that

$$
\begin{aligned}
\left\|z_{n+1}-z_{n}\right\| & \leq\left\|T_{\rho_{n+1}} w_{n+1}-T_{\rho_{n+1}} w_{n}\right\|+\left\|T_{\rho_{n+1}} w_{n}-T_{\rho_{n}} w_{n}\right\| \\
& \leq\left\|w_{n+1}-w_{n}\right\|+\left\|T_{\rho_{n+1}} w_{n}-T_{\rho_{n}} w_{n}\right\| .
\end{aligned}
$$


From Lemma 6, we know that $I-\rho \Phi$ is nonexpansive for all $\rho \in(0,2 \varsigma)$. Thus, we have that $I-\left(\left(\rho_{n+1}\right) /\left(1-\varsigma_{n+1}\right)\right) \Phi$ is nonexpansive for all $n$ due to the fact that $\left(\rho_{n+1}\right) /\left(1-\varsigma_{n+1}\right) \in$ $(0,2 \varsigma)$. Then, we get

$$
\begin{aligned}
& \left\|w_{n+1}-w_{n}\right\|=\|\left(1-\varsigma_{n+1}\right) x_{n+1}+\varsigma_{n+1} u_{n+1}-\rho_{n+1} \Phi\left(x_{n+1}\right) \\
& -\left(\left(1-\varsigma_{n}\right) x_{n}+\varsigma_{n} u_{n}-\rho_{n} \Phi\left(x_{n}\right)\right) \| \\
& =\|\left(1-\varsigma_{n+1}\right)\left(x_{n+1}-\frac{\rho_{n+1}}{1-\varsigma_{n+1}} \Phi\left(x_{n+1}\right)\right) \\
& -\left(1-\varsigma_{n}\right)\left(x_{n}-\frac{\rho_{n}}{1-\varsigma_{n}} \Phi\left(x_{n}\right)\right) \\
& +\left(\varsigma_{n+1}-\varsigma_{n}\right) u_{n+1}+\varsigma_{n}\left(u_{n+1}-u_{n}\right) \| \\
& \leq\left(1-\varsigma_{n+1}\right) \|\left(I-\frac{\rho_{n+1}}{1-\varsigma_{n+1}} \Phi\right) x_{n+1} \\
& -\left(I-\frac{\rho_{n+1}}{1-\varsigma_{n+1}} \Phi\right) x_{n} \| \\
& +\|\left(1-\varsigma_{n+1}\right) x_{n}-\rho_{n+1} \Phi\left(x_{n}\right) \\
& -\left(1-\varsigma_{n}\right) x_{n}+\rho_{n} \Phi\left(x_{n}\right) \| \\
& +\left|\varsigma_{n+1}-\varsigma_{n}\right|\left\|u_{n+1}\right\|+\varsigma_{n}\left\|u_{n+1}-u_{n}\right\| \\
& \leq\left\|x_{n+1}-x_{n}\right\|+\left|\varsigma_{n+1}-\varsigma_{n}\right|\left(\left\|x_{n}\right\|+\left\|u_{n+1}\right\|\right) \\
& +\varsigma_{n}\left\|u_{n+1}-u_{n}\right\|+\left|\rho_{n+1}-\rho_{n}\right|\left\|\Phi\left(x_{n}\right)\right\| \text {. }
\end{aligned}
$$

By Lemma 5, we have

$$
\left\|T_{\rho_{n+1}} w_{n}-T_{\rho_{n}} w_{n}\right\| \leq \frac{\left|\rho_{n+1}-\rho_{n}\right|}{\rho_{n+1}}\left\|T_{\rho_{n+1}} w_{n}-w_{n}\right\| .
$$

From (18)-(20), we obtain

$$
\begin{aligned}
\left\|z_{n+1}-z_{n}\right\| \leq & \left\|x_{n+1}-x_{n}\right\|+\left|\varsigma_{n+1}-\varsigma_{n}\right|\left(\left\|x_{n}\right\|+\left\|u_{n+1}\right\|\right) \\
& +\varsigma_{n}\left\|u_{n+1}-u_{n}\right\|+\left|\rho_{n+1}-\rho_{n}\right|\left\|\Phi\left(x_{n}\right)\right\| \\
& +\frac{\left|\rho_{n+1}-\rho_{n}\right|}{\rho_{n+1}}\left\|T_{\rho_{n+1}} w_{n}-w_{n}\right\| .
\end{aligned}
$$

Then,

$$
\begin{aligned}
\left\|S z_{n+1}-S z_{n}\right\| \leq & \left\|z_{n+1}-z_{n}\right\| \\
\leq & \left\|x_{n+1}-x_{n}\right\|+\left|\varsigma_{n+1}-\varsigma_{n}\right| \\
& \times\left(\left\|x_{n}\right\|+\left\|u_{n+1}\right\|\right)+\varsigma_{n}\left\|u_{n+1}-u_{n}\right\| \\
& +\left|\rho_{n+1}-\rho_{n}\right|\left\|\Phi\left(x_{n}\right)\right\| \\
& +\frac{\left|\rho_{n+1}-\rho_{n}\right|}{\rho_{n+1}}\left\|T_{\rho_{n+1}} w_{n}-w_{n}\right\| .
\end{aligned}
$$

Therefore,

$$
\begin{aligned}
\left\|S z_{n+1}-S z_{n}\right\|-\left\|x_{n+1}-x_{n}\right\| \leq & \left|\varsigma_{n+1}-\varsigma_{n}\right|\left(\left\|x_{n}\right\|+\left\|u_{n+1}\right\|\right) \\
& +\varsigma_{n}\left\|u_{n+1}-u_{n}\right\| \\
& +\left|\rho_{n+1}-\rho_{n}\right|\left\|\Phi\left(x_{n}\right)\right\| \\
& +\frac{\left|\rho_{n+1}-\rho_{n}\right|}{\rho_{n+1}}\left\|T_{\rho_{n+1}} w_{n}-w_{n}\right\| .
\end{aligned}
$$

Since $\varsigma_{n} \rightarrow 0, \rho_{n+1}-\rho_{n} \rightarrow 0$ and $\liminf _{n \rightarrow \infty} \rho_{n}>0$, we obtain

$$
\limsup _{n \rightarrow \infty}\left(\left\|S z_{n+1}-S z_{n}\right\|-\left\|x_{n+1}-x_{n}\right\|\right) \leq 0 .
$$

By Lemma 8, we get

$$
\lim _{n \rightarrow \infty}\left\|S z_{n}-x_{n}\right\|=0
$$

Consequently, we obtain

$$
\lim _{n \rightarrow \infty}\left\|x_{n+1}-x_{n}\right\|=\lim _{n \rightarrow \infty}\left(1-\oplus_{n}\right)\left\|S z_{n}-x_{n}\right\|=0 .
$$

From (11) and (14), we have

$$
\begin{aligned}
\left\|x_{n+1}-z\right\|^{2} \leq & \omega_{n}\left\|x_{n}-z\right\|^{2}+\left(1-\Phi_{n}\right) \\
& \times\left\|S T_{\rho_{n}}\left[\left(1-\varsigma_{n}\right) x_{n}+\varsigma_{n} u_{n}-\rho_{n} \Phi\left(x_{n}\right)\right]-z\right\|^{2} \\
\leq & \left(1-\Phi_{n}\right)\left\{( 1 - \varsigma _ { n } ) \left(\left\|x_{n}-z\right\|^{2}+\frac{\rho_{n}}{\left(1-\varsigma_{n}\right)^{2}}\right.\right. \\
& \times\left(\rho_{n}-2\left(1-\varsigma_{n}\right) \varsigma\right) \\
& \left.\times\left\|\Phi\left(x_{n}\right)-\Phi(z)\right\|^{2}\right) \\
= & {\left.\left[1-\left(1-\varsigma_{n}\right) \varsigma_{n}\right]\left\|u_{n}-z\right\|_{n}^{2}\right\}+\omega^{2}+\frac{\left(1-\Phi_{n}\right) \rho_{n}}{1-\varsigma_{n}} } \\
& \times\left(\rho_{n}-2\left(1-\varsigma_{n}\right) \varsigma\right)\left\|\Phi\left(x_{n}\right)-\Phi(z)\right\|^{2} \\
& +\left(1-\Phi_{n}\right) \varsigma_{n}\left\|u_{n}-z\right\|^{2} \\
\leq & \left\|x_{n}-z\right\|^{2}+\frac{\left(1-\Phi_{n}\right) \rho_{n}}{1-\varsigma_{n}}\left(\rho_{n}-2\left(1-\varsigma_{n}\right) \varsigma\right) \\
& \times\left\|\Phi\left(x_{n}\right)-\Phi(z)\right\|^{2}+\left(1-\Phi_{n}\right) \varsigma_{n}\left\|u_{n}-z\right\|^{2} .
\end{aligned}
$$


Then, we obtain

$$
\begin{aligned}
& \frac{\left(1-\Theta_{n}\right) \rho_{n}}{1-\varsigma_{n}}\left(2\left(1-\varsigma_{n}\right) \varsigma-\rho_{n}\right)\left\|\Phi\left(x_{n}\right)-\Phi(z)\right\|^{2} \\
& \leq\left\|x_{n}-z\right\|^{2}-\left\|x_{n+1}-z\right\|^{2}+\left(1-\varrho_{n}\right) \varsigma_{n}\left\|u_{n}-z\right\|^{2} \\
& \leq\left(\left\|x_{n}-z\right\|-\left\|x_{n+1}-z\right\|\right)\left\|x_{n+1}-x_{n}\right\| \\
& \quad+\left(1-\varrho_{n}\right) \varsigma_{n}\left\|u_{n}-z\right\|^{2} .
\end{aligned}
$$

Since $\lim _{n \rightarrow \infty} \varsigma_{n}=0, \lim _{n \rightarrow \infty}\left\|x_{n+1}-x_{n}\right\|=0$, and $\liminf _{n \rightarrow \infty}\left(\left(1-\omega_{n}\right) \rho_{n} /\left(1-\varsigma_{n}\right)\right)\left(2\left(1-\varsigma_{n}\right) \varsigma-\rho_{n}\right)>0$, we have

$$
\lim _{n \rightarrow \infty}\left\|\Phi\left(x_{n}\right)-\Phi(z)\right\|=0
$$

Next, we show $\left\|x_{n}-T_{\rho_{n}} w_{n}\right\| \rightarrow 0$. By using the firm nonexpansivity of $T_{\rho_{n}}$, we have

$$
\begin{aligned}
\left\|T_{\rho_{n}} w_{n}-z\right\|^{2} & =\left\|T_{\rho_{n}} w_{n}-T_{\rho_{n}}\left(z-\rho_{n} \Phi(z)\right)\right\|^{2} \\
\leq & \left\langle w_{n}-\left(z-\rho_{n} \Phi(z)\right), T_{\rho_{n}} w_{n}-z\right\rangle \\
= & \frac{1}{2}\left(\left\|w_{n}-\left(z-\rho_{n} \Phi(z)\right)\right\|^{2}+\left\|T_{\rho_{n}} w_{n}-z\right\|^{2}\right. \\
& -\|\left(1-\varsigma_{n}\right) x_{n}+\varsigma_{n} u_{n} \\
& \left.\quad-\rho_{n}\left(\Phi\left(x_{n}\right)-\Phi(z)\right)-T_{\rho_{n}} w_{n} \|^{2}\right) .
\end{aligned}
$$

We note that

$$
\left\|w_{n}-\left(z-\rho_{n} \Phi(z)\right)\right\|^{2} \leq\left(1-\varsigma_{n}\right)\left\|x_{n}-z\right\|^{2}+\varsigma_{n}\left\|u_{n}-z\right\|^{2} .
$$

Thus,

$$
\begin{aligned}
\left\|T_{\rho_{n}} w_{n}-z\right\|^{2} \leq \frac{1}{2}( & \left(1-\varsigma_{n}\right)\left\|x_{n}-z\right\|^{2} \\
+ & \varsigma_{n}\left\|u_{n}-z\right\|^{2}+\left\|T_{\rho_{n}} w_{n}-z\right\|^{2} \\
- & \|\left(1-\varsigma_{n}\right) x_{n}+\varsigma_{n} u_{n}-T_{\rho_{n}} w_{n} \\
& \left.-\rho_{n}\left(\Phi\left(x_{n}\right)-\Phi(z)\right) \|^{2}\right) ;
\end{aligned}
$$

that is,

$$
\begin{array}{r}
\left\|T_{\rho_{n}} w_{n}-z\right\|^{2} \leq\left(1-\varsigma_{n}\right)\left\|x_{n}-z\right\|^{2}+\varsigma_{n}\left\|u_{n}-z\right\|^{2} \\
-\|\left(1-\varsigma_{n}\right) x_{n}+\varsigma_{n} u_{n}-T_{\rho_{n}} w_{n} \\
-\rho_{n}\left(\Phi\left(x_{n}\right)-\Phi(z)\right) \|^{2}
\end{array}
$$

$$
\begin{aligned}
& =\left(1-\varsigma_{n}\right)\left\|x_{n}-z\right\|^{2}+\varsigma_{n}\left\|u_{n}-z\right\|^{2} \\
& -\left\|\left(1-\varsigma_{n}\right) x_{n}+\varsigma_{n} u_{n}-T_{\rho_{n}} w_{n}\right\|^{2} \\
& +2 \rho_{n}\left\langle\left(1-\varsigma_{n}\right) x_{n}+\varsigma_{n} u_{n}-T_{\rho_{n}} w_{n}, \Phi\left(x_{n}\right)\right. \\
& \quad-\Phi(z)\rangle-\rho_{n}^{2}\left\|\Phi\left(x_{n}\right)-\Phi(z)\right\|^{2} \\
& \leq\left(1-\varsigma_{n}\right)\left\|x_{n}-z\right\|^{2}+\varsigma_{n}\left\|u_{n}-z\right\|^{2} \\
& \quad-\left\|\left(1-\varsigma_{n}\right) x_{n}+\varsigma_{n} u_{n}-T_{\rho_{n}} w_{n}\right\|^{2} \\
& +2 \rho_{n}\left\|\left(1-\varsigma_{n}\right) x_{n}+\varsigma_{n} u_{n}-T_{\rho_{n}} w_{n}\right\| \\
& \quad \times\left\|\Phi\left(x_{n}\right)-\Phi(z)\right\| .
\end{aligned}
$$

It follows that

$$
\begin{aligned}
& \left\|x_{n+1}-z\right\|^{2} \leq \omega_{n}\left\|x_{n}-z\right\|^{2}+\left(1-\omega_{n}\right)\left(1-\varsigma_{n}\right) \\
& \times\left\|x_{n}-z\right\|^{2}+\left(1-\omega_{n}\right) \varsigma_{n}\left\|u_{n}-z\right\|^{2} \\
& -\left(1-\omega_{n}\right)\left\|\left(1-\varsigma_{n}\right) x_{n}+\varsigma_{n} u_{n}-T_{\rho_{n}} w_{n}\right\|^{2} \\
& +2 \rho_{n}\left(1-\omega_{n}\right)\left\|\left(1-\varsigma_{n}\right) x_{n}+\varsigma_{n} u_{n}-T_{\rho_{n}} w_{n}\right\| \\
& \times\left\|\Phi\left(x_{n}\right)-\Phi(z)\right\| \\
& =\left[1-\left(1-\omega_{n}\right) \varsigma_{n}\right]\left\|x_{n}-z\right\|^{2} \\
& +\left(1-\omega_{n}\right) \varsigma_{n}\left\|u_{n}-z\right\|^{2}-\left(1-\omega_{n}\right) \\
& \times\left\|\left(1-\varsigma_{n}\right) x_{n}+\varsigma_{n} u_{n}-T_{\rho_{n}} w_{n}\right\|^{2} \\
& +2 \rho_{n}\left(1-\omega_{n}\right)\left\|\left(1-\varsigma_{n}\right) x_{n}+\varsigma_{n} u_{n}-T_{\rho_{n}} w_{n}\right\| \\
& \times\left\|\Phi\left(x_{n}\right)-\Phi(z)\right\| \text {. }
\end{aligned}
$$

Hence,

$$
\begin{aligned}
\left(1-\omega_{n}\right) & \left\|\left(1-\varsigma_{n}\right) x_{n}+\varsigma_{n} u_{n}-T_{\rho_{n}} w_{n}\right\|^{2} \\
\leq & \left\|x_{n}-z\right\|^{2}-\left\|x_{n+1}-z\right\|^{2} \\
- & \left(1-\omega_{n}\right) \varsigma_{n}\left\|x_{n}-z\right\|^{2} \\
+ & \left(1-\varrho_{n}\right) \varsigma_{n}\left\|u_{n}-z\right\|^{2}+2 \rho_{n}\left(1-\varrho_{n}\right) \\
& \times\left\|\left(1-\varsigma_{n}\right) x_{n}+\varsigma_{n} u_{n}-T_{\rho_{n}} w_{n}\right\|\left\|\Phi\left(x_{n}\right)-\Phi(z)\right\| \\
\leq & \left(\left\|x_{n}-z\right\|+\left\|x_{n+1}-z\right\|\right)\left\|x_{n+1}-x_{n}\right\| \\
+ & \left(1-\omega_{n}\right) \varsigma_{n}\left\|u_{n}-z\right\|^{2}+2 \rho_{n}\left(1-\Phi_{n}\right) \\
\quad & \times\left(1-\varsigma_{n}\right) x_{n}+\varsigma_{n} u_{n}-T_{\rho_{n}} w_{n}\|\| \Phi\left(x_{n}\right)-\Phi(z) \| .
\end{aligned}
$$


Since $\lim \sup _{n \rightarrow \infty} \omega_{n}<1,\left\|x_{n+1}-x_{n}\right\| \rightarrow 0, \varsigma_{n} \rightarrow 0$, and $\left\|\Phi\left(x_{n}\right)-\Phi(z)\right\| \rightarrow 0$, we deduce

$$
\lim _{n \rightarrow \infty}\left\|\left(1-\varsigma_{n}\right) x_{n}+\varsigma_{n} u_{n}-T_{\rho_{n}} w_{n}\right\|=0
$$

This implies that

$$
\lim _{n \rightarrow \infty}\left\|x_{n}-T_{\rho_{n}} w_{n}\right\|=\lim _{n \rightarrow \infty}\left\|x_{n}-z_{n}\right\|=0 .
$$

Conclusion 3. Consider

$$
\limsup _{n \rightarrow \infty}\left\langle\tilde{x}-u, x_{n}-\tilde{x}\right\rangle \geq 0,
$$

where $\tilde{x}=P_{F(S) \cap E P}(u)$.

Proof. Since $\left\{x_{n}\right\}$ is bounded, there exists a subsequence $\left\{x_{n_{i}}\right\}$ of $\left\{x_{n}\right\}$ such that $x_{n_{i}} \rightarrow w$ weakly and

$$
\limsup _{n \rightarrow \infty}\left\langle\tilde{x}-u, x_{n}-\tilde{x}\right\rangle=\lim _{i \rightarrow \infty}\left\langle\tilde{x}-u, x_{n_{i}}-\tilde{x}\right\rangle .
$$

By (25) and (37), we deduce

$$
\lim _{n \rightarrow \infty}\left\|x_{n}-S x_{n}\right\|=0 .
$$

Hence,

$$
\lim _{i \rightarrow \infty}\left\|x_{n_{i}}-S x_{n_{i}}\right\|=0 .
$$

This together with Lemma 7 implies that $w \in F(S)$.

Next we show that $\tilde{x} \in$ EP. Since $z_{n}=T_{\rho_{n}}\left[\left(1-\varsigma_{n}\right) x_{n}+\right.$ $\left.\varsigma_{n} u_{n}-\rho_{n} \Phi\left(x_{n}\right)\right]$ for any $y \in C$, we have

$$
\begin{aligned}
\Theta\left(z_{n}, y\right) & +\left\langle\Phi\left(x_{n}\right), y-z_{n}\right\rangle \\
& +\frac{1}{\rho_{n}}\left\langle y-z_{n}, z_{n}-\left(1-\varsigma_{n}\right) x_{n}-\varsigma_{n} u_{n}\right\rangle \geq 0 .
\end{aligned}
$$

From (C2), we have

$$
\begin{aligned}
\left\langle\Phi\left(x_{n}\right), y-z_{n}\right\rangle & +\frac{1}{\rho_{n}}\left\langle y-z_{n}, z_{n}-\left(1-\varsigma_{n}\right) x_{n}-\varsigma_{n} u_{n}\right\rangle \\
& \geq \Theta\left(y, z_{n}\right) .
\end{aligned}
$$

Put $x_{t}=t y+(1-t) \tilde{x}$ for all $t \in(0,1)$ and $y \in C$. Then, we have $x_{t} \in C$. So, from (43), we have

$$
\begin{aligned}
\left\langle x_{t}-z_{n}, \Phi\left(x_{t}\right)\right\rangle \geq & \left\langle x_{t}-z_{n}, \Phi\left(x_{t}\right)\right\rangle-\left\langle\Phi\left(x_{n}\right), x_{t}-z_{n}\right\rangle \\
& -\frac{1}{\rho_{n}}\left\langle x_{t}-z_{n}, z_{n}-\left(1-\varsigma_{n}\right) x_{n}-\varsigma_{n} u_{n}\right\rangle \\
& +\Theta\left(x_{t}, z_{n}\right) \\
= & \left\langle x_{t}-z_{n}, \Phi\left(x_{t}\right)-\Phi\left(z_{n}\right)\right\rangle \\
& +\left\langle x_{t}-z_{n}, \Phi\left(z_{n}\right)-\Phi\left(x_{n}\right)\right\rangle \\
& -\frac{1}{\rho_{n}}\left\langle x_{t}-z_{n}, z_{n}-\left(1-\varsigma_{n}\right) x_{n}-\varsigma_{n} u_{n}\right\rangle \\
& +\Theta\left(x_{t}, z_{n}\right) .
\end{aligned}
$$

Since $\Phi$ is $\varsigma$-inverse-strongly monotone, $\Phi$ is $(1 / \varsigma)$ Lipschitzian. By (37), we derive that $\left\|\Phi\left(z_{n}\right)-\Phi\left(x_{n}\right)\right\| \rightarrow 0$. Further, from monotonicity of $\Phi$, we have $\left\langle x_{t}-z_{n}, \Phi\left(x_{t}\right)-\right.$ $\left.\Phi\left(z_{n}\right)\right\rangle \geq 0$. Letting $n \rightarrow \infty$ in (45) and noting (C4), we have

$$
\left\langle x_{t}-\tilde{x}, \Phi\left(x_{t}\right)\right\rangle \geq \Theta\left(x_{t}, \tilde{x}\right) .
$$

By (C1), (C4), and (45), we deduce

$$
\begin{aligned}
0 & =\Theta\left(x_{t}, x_{t}\right) \\
& \leq t \Theta\left(x_{t}, y\right)+(1-t) \Theta\left(x_{t}, \tilde{x}\right) \\
& \leq t \Theta\left(x_{t}, y\right)+(1-t)\left\langle x_{t}-\tilde{x}, \Phi\left(x_{t}\right)\right\rangle \\
& =t \Theta\left(x_{t}, y\right)+(1-t) t\left\langle y-\tilde{x}, \Phi\left(x_{t}\right)\right\rangle,
\end{aligned}
$$

and hence

$$
0 \leq \Theta\left(x_{t}, y\right)+(1-t)\left\langle y-\tilde{x}, \Phi\left(x_{t}\right)\right\rangle
$$

Letting $t \rightarrow 0$, we have, for each $y \in C$,

$$
0 \leq \Theta(\tilde{x}, y)+\langle y-\tilde{x}, \Phi(\tilde{x})\rangle .
$$

This implies $\tilde{x} \in \mathrm{EP}$. Therefore, we have $w \in F(S) \cap \mathrm{EP}$. So,

$$
\begin{aligned}
\limsup _{n \rightarrow \infty}\left\langle\tilde{x}-u, x_{n}-\tilde{x}\right\rangle & =\lim _{i \rightarrow \infty}\left\langle\tilde{x}-u, x_{n_{i}}-\tilde{x}\right\rangle \\
& =\langle\tilde{x}-u, w-\tilde{x}\rangle \geq 0 .
\end{aligned}
$$

Setting $v_{n}=x_{n}-\left(\rho_{n} /\left(1-\varsigma_{n}\right)\right)\left(\Phi\left(x_{n}\right)-\Phi(\tilde{x})\right)$ for all $n$ and taking $z=\tilde{x}$ in (29) to get $\left\|\Phi\left(x_{n}\right)-\Phi(\tilde{x})\right\| \rightarrow 0$, so, $v_{n}-x_{n} \rightarrow$ 0 . Since $u_{n} \rightarrow u$, we have

$$
\begin{aligned}
\limsup _{n \rightarrow \infty}\left\langle u_{n}-\tilde{x}, v_{n}-\tilde{x}\right\rangle & =\limsup _{n \rightarrow \infty}\left\langle u-\tilde{x}, v_{n}-\tilde{x}\right\rangle \\
& =\limsup _{n \rightarrow \infty}\left\langle u-\tilde{x}, x_{n}-\tilde{x}\right\rangle \leq 0 .
\end{aligned}
$$

Conclusion $4\left(x_{n} \rightarrow \tilde{x}\right)$.

Proof. From (11), we have

$$
\begin{aligned}
\left\|x_{n+1}-\tilde{x}\right\|^{2} \leq & \omega_{n}\left\|x_{n}-\tilde{x}\right\|^{2}+\left(1-\omega_{n}\right)\left\|S T_{\rho_{n}} w_{n}-\tilde{x}\right\|^{2} \\
\leq & \omega_{n}\left\|x_{n}-\tilde{x}\right\|^{2}+\left(1-\omega_{n}\right)\left\|T_{\rho_{n}} w_{n}-\tilde{x}\right\|^{2} \\
= & \omega_{n}\left\|x_{n}-\tilde{x}\right\|^{2}+\left(1-\omega_{n}\right) \\
& \times\left\|T_{\rho_{n}} w_{n}-T_{\rho_{n}}\left(\tilde{x}-\rho_{n} \Phi(\tilde{x})\right)\right\|^{2}
\end{aligned}
$$




$$
\begin{aligned}
& \leq \omega_{n}\left\|x_{n}-\tilde{x}\right\|^{2}+\left(1-\omega_{n}\right)\left\|w_{n}-\left(\tilde{x}-\rho_{n} \Phi(\tilde{x})\right)\right\|^{2} \\
& =\omega_{n}\left\|x_{n}-\tilde{x}\right\|^{2}+\left(1-\omega_{n}\right) \\
& \times\left\|\left(1-\varsigma_{n}\right) x_{n}+\varsigma_{n} u_{n}-\rho_{n} \Phi\left(x_{n}\right)-\left(\tilde{x}-\rho_{n} \Phi(\tilde{x})\right)\right\|^{2} \\
& =\left(1-\omega_{n}\right) \|\left(1-\varsigma_{n}\right)\left(\left(x_{n}-\frac{\rho_{n}}{1-\varsigma_{n}} \Phi\left(x_{n}\right)\right)\right. \\
& \left.-\left(\tilde{x}-\frac{\rho_{n}}{1-\varsigma_{n}} \Phi(\tilde{x})\right)\right) \\
& +\varsigma_{n}\left(u_{n}-\tilde{x}\right) \|^{2} \\
& +\omega_{n}\left\|x_{n}-\tilde{x}\right\|^{2} \\
& =\left(1-\omega_{n}\right)\left(\left(1-\varsigma_{n}\right)^{2} \|\left(x_{n}-\frac{\rho_{n}}{1-\varsigma_{n}} \Phi\left(x_{n}\right)\right)\right. \\
& -\left(\tilde{x}-\frac{\rho_{n}}{1-\varsigma_{n}} \Phi(\tilde{x})\right) \|^{2} \\
& +2 \varsigma_{n}\left(1-\varsigma_{n}\right) \\
& \times\left\langle u_{n}-\tilde{x},\left(x_{n}-\frac{\rho_{n}}{1-\varsigma_{n}} \Phi\left(x_{n}\right)\right)\right. \\
& \left.-\left(\tilde{x}-\frac{\rho_{n}}{1-\varsigma_{n}} \Phi(\tilde{x})\right)\right\rangle \\
& \left.+\varsigma_{n}^{2}\left\|u_{n}-\tilde{x}\right\|^{2}\right) \\
& +\omega_{n}\left\|x_{n}-\tilde{x}\right\|^{2} \\
& \leq \omega_{n}\left\|x_{n}-\tilde{x}\right\|^{2}+\left(1-\omega_{n}\right) \\
& \times\left(\left(1-\varsigma_{n}\right)^{2}\left\|x_{n}-\tilde{x}\right\|^{2}+2 \varsigma_{n}\left(1-\varsigma_{n}\right)\right. \\
& \times\left\langle u_{n}-\tilde{x}, x_{n}-\frac{\rho_{n}}{1-\varsigma_{n}}\right. \\
& \times\left(\Phi\left(x_{n}\right)-\Phi(\tilde{x})\right) \\
& -\tilde{x}\rangle \\
& \left.+\varsigma_{n}^{2}\left\|u_{n}-\tilde{x}\right\|^{2}\right) \\
& \leq\left[1-\left(1-\omega_{n}\right) \varsigma_{n}\right]\left\|x_{n}-\tilde{x}\right\|^{2}+\left(1-\omega_{n}\right) \varsigma_{n} \\
& \times\left\{2\left(1-\varsigma_{n}\right)\left\langle u_{n}-\tilde{x}, v_{n}-\tilde{x}\right\rangle+\varsigma_{n}\left\|u_{n}-\tilde{x}\right\|^{2}\right\} .
\end{aligned}
$$

It is clear that $\sum_{n=1}^{\infty}\left(1-\omega_{n}\right) \varsigma_{n}=\infty$ and $\lim \sup _{n \rightarrow \infty}(2(1-$ $\left.\left.\varsigma_{n}\right)\left\langle u_{n}-\tilde{x}, v_{n}-\tilde{x}\right\rangle+\varsigma_{n}\left\|u_{n}-\tilde{x}\right\|^{2}\right) \leq 0$. We can therefore apply Lemma 9 to conclude that $x_{n} \rightarrow \tilde{x}$. This completes the proof.
Corollary 11. Let $C$ be a nonempty closed convex subset of $H$ and let $\Theta: C \times C \rightarrow R$ be a bifunction satisfying conditions (C1)-(C4). Let $\Phi: C \rightarrow H$ be an $\varsigma$-inverse-strongly monotone mapping and let $S: C \rightarrow C$ be a nonexpansive mapping. Suppose that $F(S) \cap E P \neq \emptyset$. For $x_{0} \in C$ arbitrarily, let the sequence $\left\{x_{n}\right\}$ be generated by

$$
x_{n+1}=\omega_{n} x_{n}+\left(1-\omega_{n}\right) S T_{\rho_{n}}\left[\left(1-\varsigma_{n}\right) x_{n}-\rho_{n} \Phi\left(x_{n}\right)\right], \quad n \geq 0,
$$

where $T_{\rho_{n}}$ is defined as that in Lemma 4 and $\left\{\rho_{n}\right\} \quad \subset$ $(0,2 \varsigma),\left\{\varsigma_{n}\right\} \subset(0,1)$, and $\left\{\omega_{n}\right\} \subset(0,1)$ satisfy

(i) $\lim _{n \rightarrow \infty} \varsigma_{n}=0$ and $\sum_{n=1}^{\infty} \varsigma_{n}=\infty$;

(ii) $0<c \leq \omega_{n} \leq d<1$;

(iii) $a\left(1-\varsigma_{n}\right) \leq \rho_{n} \leq b\left(1-\varsigma_{n}\right)$, where $[a, b] \subset(0,2 \varsigma)$ and $\lim _{n \rightarrow \infty}\left(\rho_{n+1}-\rho_{n}\right)=0$.

Then $\left\{x_{n}\right\}$ generated by (52) converges strongly to $P_{F(S) \cap E P}(0)$ which is the minimum norm element in $F(S) \cap E P$.

\section{Conflict of Interests}

The authors declare that there is no conflict of interests regarding the publication of this paper.

\section{Acknowledgment}

This study was supported by research funds from Dong-A University.

\section{References}

[1] E. Blum and W. Oettli, "From optimization and variational inequalities to equilibrium problems," The Mathematics Student, vol. 63, no. 1-4, pp. 123-145, 1994.

[2] L.-C. Ceng, Q. H. Ansari, and S. Schaible, "Hybrid extragradient-like methods for generalized mixed equilibrium problems, systems of generalized equilibrium problems and optimization problems," Journal of Global Optimization. An International Journal Dealing with Theoretical and Computational Aspects of Seeking Global Optima and Their Applications in Science, Management and Engineering, vol. 53, no. 1, pp. 69-96, 2012.

[3] L.-C. Ceng, S.-M. Guu, and J.-C. Yao, "Hybrid iterative method for finding common solutions of generalized mixed equilibrium and fixed point problems," Fixed Point Theory and Applications, vol. 92, p. 2012, article 19, 2012.

[4] L. C. Ceng, H.-Y. Hu, and M. M. Wong, "Strong and weak convergence theorems for generalized mixed equilibrium problem with perturbation and fixed pointed problem of infinitely many nonexpansive mappings," Taiwanese Journal of Mathematics, vol. 15, no. 3, pp. 1341-1367, 2011.

[5] L. C. Ceng, A. Petruşel, and J. C. Yao, "Iterative approaches to solving equilibrium problems and fixed point problems of infinitely many nonexpansive mappings," Journal of Optimization Theory and Applications, vol. 143, no. 1, pp. 37-58, 2009.

[6] L. C. Ceng, A. Petrușel, and J. C. Yao, "Iterative approaches to solving equilibrium problems and fixed point problems of infinitely many nonexpansive mappings," Journal of Optimization Theory and Applications, vol. 143, no. 1, pp. 37-58, 2009. 
[7] L. C. Ceng, S. Schaible, and J. C. Yao, "Implicit iteration scheme with perturbed mapping for equilibrium problems and fixed point problems of finitely many nonexpansive mappings," Journal of Optimization Theory and Applications, vol. 139, no. 2, pp. 403-418, 2008.

[8] L.-C. Ceng and J.-C. Yao, "A hybrid iterative scheme for mixed equilibrium problems and fixed point problems," Journal of Computational and Applied Mathematics, vol. 214, no. 1, pp. 186201, 2008.

[9] L.-C. Ceng and J.-C. Yao, "Hybrid viscosity approximation schemes for equilibrium problems and fixed point problems of infinitely many nonexpansive mappings," Applied Mathematics and Computation, vol. 198, no. 2, pp. 729-741, 2008.

[10] L.-C. Ceng and J.-C. Yao, "A relaxed extragradient-like method for a generalized mixed equilibrium problem, a general system of generalized equilibria and a fixed point problem," Nonlinear Analysis. Theory, Methods \& Applications. An International Multidisciplinary Journal A: Theory and Methods, vol. 72, no. 34, pp. 1922-1937, 2010.

[11] P. L. Combettes and S. A. Hirstoaga, "Equilibrium programming in Hilbert spaces," Journal of Nonlinear and Convex Analysis. An International Journal, vol. 6, no. 1, pp. 117-136, 2005.

[12] A. Moudafi, "Weak convergence theorems for nonexpansive mappings and equilibrium problems," Journal of Nonlinear and Convex Analysis. An International Journal, vol. 9, no. 1, pp. 3743, 2008.

[13] A. Moudafi and M. Thera, "Proximal and dynamical approaches to equilibrium problems," in Lecture Notes in Economics and Mathematical Systems, vol. 477, pp. 187-201, Springer, 1999.

[14] J.-W. Peng and J.-C. Yao, "A new hybrid-extragradient method for generalized mixed equilibrium problems, fixed point problems and variational inequality problems," Taiwanese Journal of Mathematics, vol. 12, no. 6, pp. 1401-1432, 2008.

[15] J.-W. Peng and J.-C. Yao, "Some new iterative algorithms for generalized mixed equilibrium problems with strict pseudocontractions and monotone mappings," Taiwanese Journal of Mathematics, vol. 13, no. 5, pp. 1537-1582, 2009.

[16] J.-W. Peng and J.-C. Yao, "Strong convergence theorems of iterative scheme based on the extragradient method for mixed equilibrium problems and fixed point problems," Mathematical and Computer Modelling, vol. 49, no. 9-10, pp. 1816-1828, 2009.

[17] S. Takahashi and W. Takahashi, "Strong convergence theorem for a generalized equilibrium problem and a nonexpansive mapping in a Hilbert space," Nonlinear Analysis. Theory, Methods \& Applications. An International Multidisciplinary Journal A: Theory and Methods, vol. 69, no. 3, pp. 1025-1033, 2008.

[18] C.-S. Chuang, L.-J. Lin, and W. Takahashi, "Halpern's type iterations with perturbations in Hilbert spaces: equilibrium solutions and fixed points," Journal of Global Optimization. An International Journal Dealing with Theoretical and Computational Aspects of Seeking Global Optima and Their Applications in Science, Management and Engineering, vol. 56, no. 4, pp. 15911601, 2013.

[19] W. Takahashi and M. Toyoda, "Weak convergence theorems for nonexpansive mappings and monotone mappings," Journal of Optimization Theory and Applications, vol. 118, no. 2, pp. 417428, 2003.

[20] K. Goebel and W. A. Kirk, Topics in Metric Fixed Point Theory, vol. 28 of Cambridge Studies in Advanced Mathematics, Cambridge University Press, Cambridge, 1990.
[21] T. Suzuki, "Strong convergence theorems for infinite families of nonexpansive mappings in general Banach spaces," Fixed Point Theory and Applications, no. 1, pp. 103-123, 2005.

[22] H.-K. Xu, "Iterative algorithms for nonlinear operators," Journal of the London Mathematical Society, vol. 66, no. 1, pp. 240-256, 2002. 


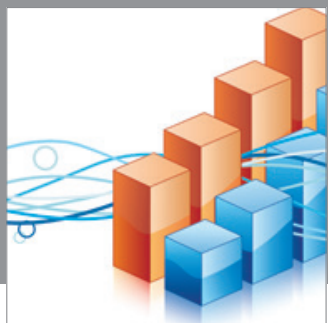

Advances in

Operations Research

mansans

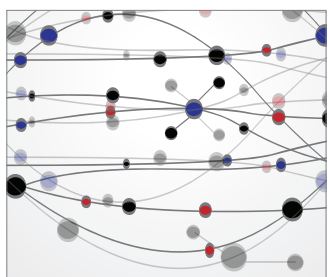

The Scientific World Journal
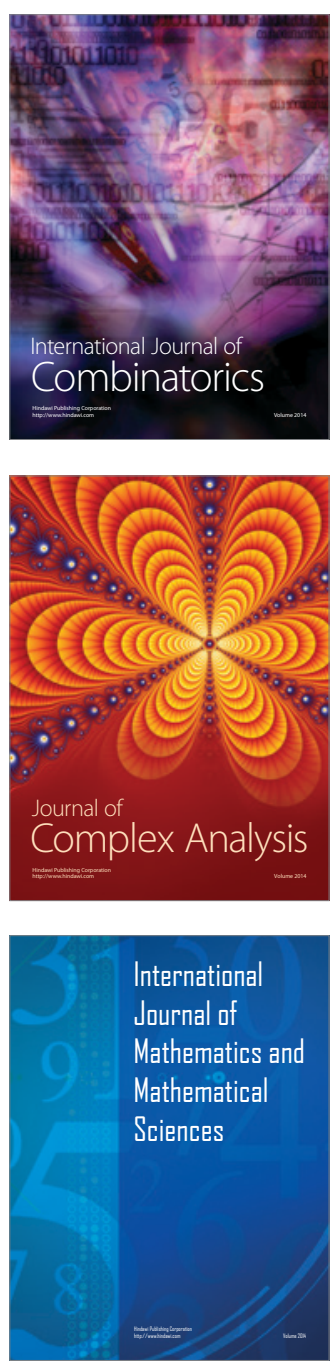
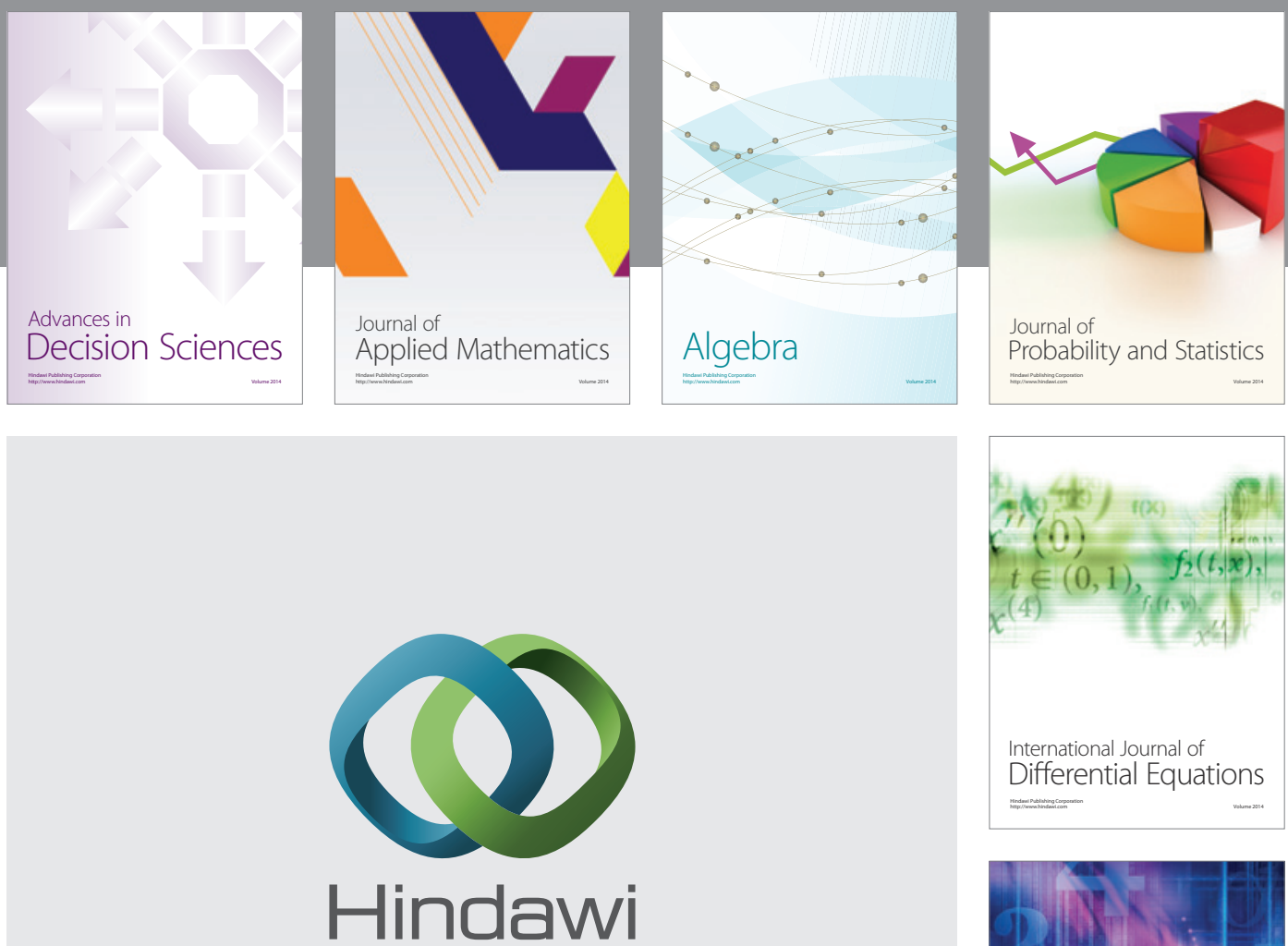

Submit your manuscripts at http://www.hindawi.com
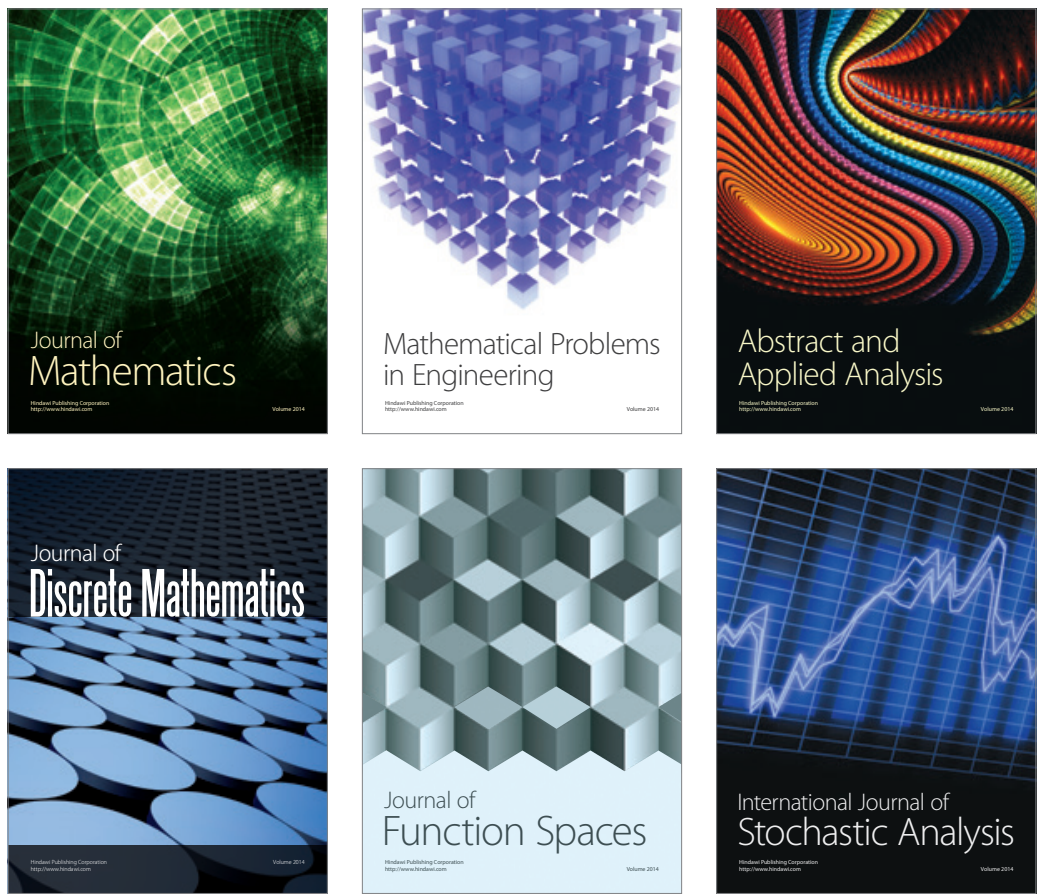

Journal of

Function Spaces

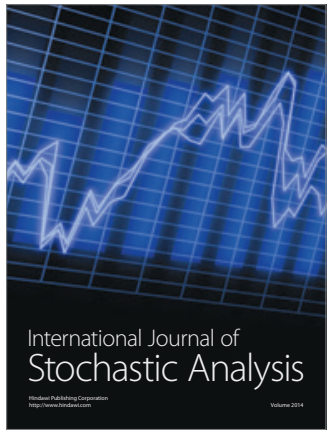

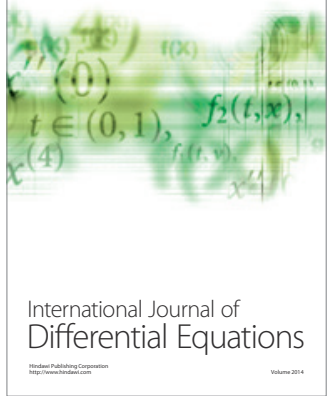
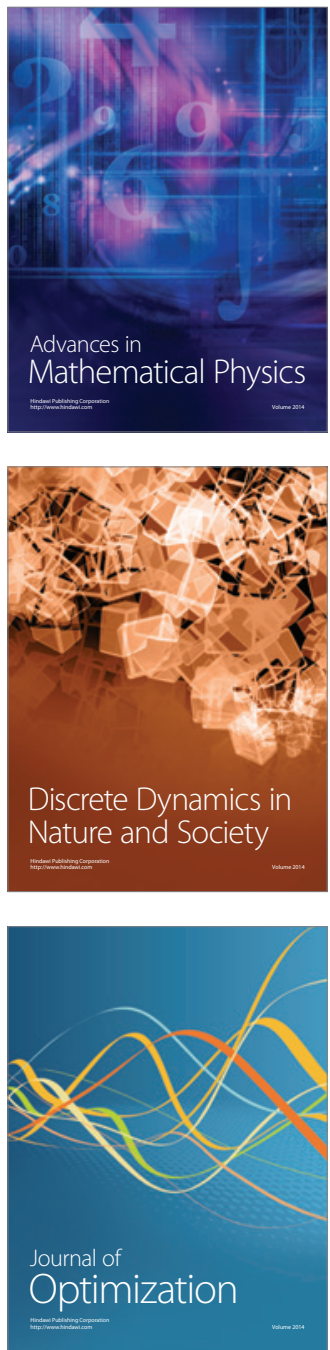